\title{
Efficient calculation of low energy statistical rates for gas phase dissociation using umbrella sampling
}

\author{
Massimo Mella ${ }^{\text {a) }}$ \\ School of Chemistry, Cardiff University, Main Building, Park Place, Cardiff CF10 3AT, United Kingdom
}

(Received 21 December 2005; accepted 27 January 2006; published online 10 March 2006)

\begin{abstract}
Monte Carlo (MC) simulations can be used to compute microcanonical statistical rates of gas phase dissociation reactions. Unfortunately, the MC approach may suffer from a slow convergence and large statistical errors for energies just above the dissociation threshold. In this work, umbrella sampling is proposed as a device to reduce the statistical error of $\mathrm{MC}$ rate constants. The method is tested by computing the classical dissociation rate for the reaction $\left[\mathrm{H}_{5} \mathrm{O}_{2}^{+}\right]^{*} \rightarrow \mathrm{H}_{2} \mathrm{O}+\mathrm{H}_{3} \mathrm{O}^{+}$over the range of internal energy $38<E \leqslant 100 \mathrm{kcal} / \mathrm{mol}$. Comparing with other literature methods, it is found that umbrella sampling reduces the computational effort by up to two orders of magnitude when used in conjunction with a careful choice of sampling distributions. The comparison between MC rate constants and classical Rice-Ramsperberg-Kassel harmonic theory shows that anharmonicity plays an important role in the dissociation process of the Zundel cation $\left(\mathrm{H}_{5} \mathrm{O}_{2}^{+}\right)$at all energies. (C) 2006 American Institute of Physics. [DOI: 10.1063/1.2178302]
\end{abstract}

A long sought goal of theoretical chemistry is the efficient calculation of reaction rates. In this respect, statistical rate theories such as transition state ${ }^{1,2}$ and RiceRamsperberg-Kassel-Marcus ${ }^{3}$ (RRKM) theories stand up as practical alternatives to the direct classical or quantum simulations of rate constants, especially when potential energy surfaces (PESs) need to be computed "on the fly.",

In the case of a system with internal energy $E$, the classical statistical rate of a process can be written as

$$
k^{\mathrm{stat}}(E)=\frac{\int_{S^{*}} \delta[\mathcal{H}(\mathbf{p}, \mathbf{q})-E] v_{\perp} d S^{*}}{\int_{V} \delta[\mathcal{H}(\mathbf{p}, \mathbf{q})-E] d \Gamma}
$$

where $S^{*}$ is the hypersurface in phase space separating the reactant from the product and $v_{\perp}$ is the velocity perpendicular to this surface. The integral at the numerator is carried out over the transition surface (state) $S^{*}$, whereas the one at the denominator is computed over the reactant phase space. Very often the dependence of $S^{*}$ on the momenta $\mathbf{p}$ is neglected, and only coordinates $\mathbf{q}$ are used for its definition.

Despite the known limitations of statistical theories (e.g., the neglect of recrossing and the assumption of fast energy exchange between modes), statistical rate constants $k^{\text {stat }}(E)$ [Eq. (1)] often provide tight upper bounds to corresponding dynamical rates $k^{\mathrm{dyn}}(E)$ computed using molecular dynamics trajectories, even for highly fluxional systems. ${ }^{6}$ This is true provided that the required integrals can be estimated numerically and that no further approximations (e.g., the harmonic one) are used to estimate the number of accessible phase space states. ${ }^{6}$

In the case of a simple bond fission process, Eq. (1) can be rewritten as

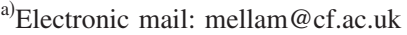

$$
k^{\mathrm{stat}}(E)=\frac{\int_{V} \delta[\mathcal{H}(\mathbf{p}, \mathbf{q})-E] \delta\left(r_{I R C}-r_{C}\right)\left|\dot{r}_{I R C}\right| d \Gamma}{\int_{V} \delta[\mathcal{H}(\mathbf{p}, \mathbf{q})-E] d \Gamma}
$$

where $r_{I R C}$ is the length of the dissociating bond, $r_{C}$ the critical distance for $r_{I R C}$, and $\dot{r}_{I R C}$ is the relative velocity of the two dissociating fragments along $r_{I R C}$. Exploiting the fact that the kinetic energy for the system is diagonal in Cartesian coordinates, the integrals in Eq. (2) can be separated and Eq. (2) can be further simplified into

$$
k^{\mathrm{stat}}(E)=\frac{\int_{\Omega}[E-\mathcal{V}(\mathbf{q})]^{(3 N-5) / 2} \delta\left(r_{I R C}-r_{C}\right)\left\langle\left|\dot{r}_{I R C}\right|\right\rangle d \mathbf{q}}{\int_{\Omega}[E-\mathcal{V}(\mathbf{q})]^{(3 N-5) / 2} d \mathbf{q}},
$$

where $\mathcal{V}(\mathbf{q})$ is the PES for the system, $N$ is the number of atoms, and $\left\langle\left|\dot{r}_{I R C}\right|\right\rangle$ is the ensemble average of the relative velocity through the critical surface. In this equation, both integrals are computed over the reactant configuration space $\Omega$. If $N$ is large, the average microcanonical value of the velocity $\left\langle\left|\dot{r}_{I R C}\right|\right\rangle$ can be accurately approximated with the corresponding canonical average $\left\langle\left|\dot{r}_{I R C}\right|\right\rangle$ $=\sqrt{4[E-\mathcal{V}(\mathbf{q})] /[3 \pi(N-1) \mu]},{ }^{8}$ where $\mu$ is the reduced mass of the two separating fragments.

Equation (3) provides three important advantages with respect to Eqs. (1) and (2). First, the dimensionality of the integrals is reduced by a factor of 2 , a substantial simplification if a numerical estimate is sought. Second, it allows the exact fulfillment of the microcanonical identity $E=\mathcal{K}+\mathcal{V}$, a requirement that needs to be somewhat relaxed in the direct sampling of the microcanonical density in phase space [Eq. (2)]. ${ }^{9}$ Third, a Monte Carlo (MC) algorithm can be used to sample $W(\mathbf{q})=[E-\mathcal{V}(\mathbf{q})]^{(3 N-5) / 2}(\mathcal{V}(\mathbf{q}) \leqslant E)$ within the range of distances $0<r_{I R C} \leqslant r_{B}\left(r_{C}<r_{B}\right)$, where $r_{B}$ is the upper limit imposed to the distance between the dissociating fragments during the MC sampling. The latter can be used to 


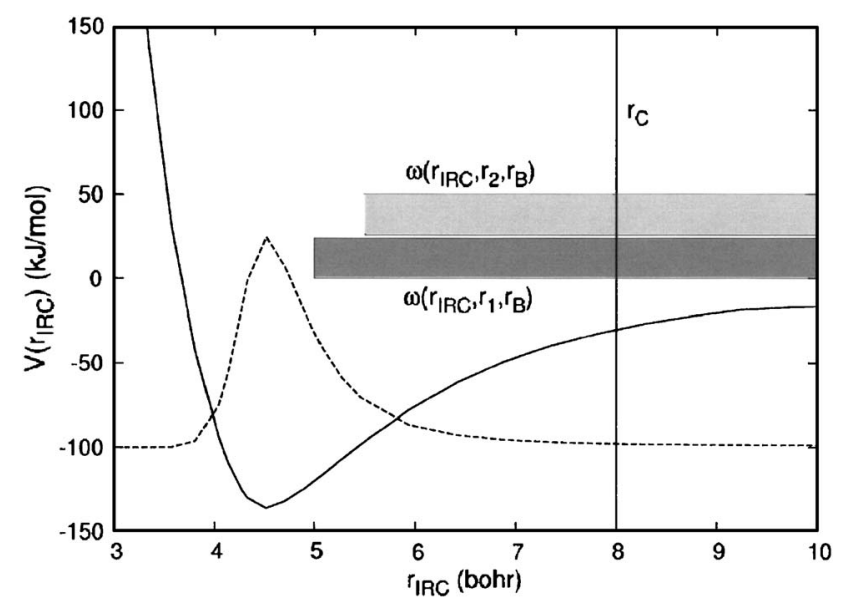

FIG. 1. Graphic representation of the method introduced by Eqs. (5) and (6). $V\left(r_{I R C}\right)$ (thick black line) is the adiabatic dissociation potential as a function of the reaction coordinate $r_{I R C}$ (i.e., the value of the potential energy when all the remaining degrees of freedom are optimized). The dashed line represents the distribution $W\left(\mathbf{R}_{I R C}\right)=\left[E-V\left(\mathbf{R}_{I R C}\right)\right]^{(3 N-5) / 2}$. The continuous vertical line represents the position of the critical distance $r_{C}$, whereas the two gray bars indicate the width of the first two windows used in the umbrella sampling approach.

estimate the average value of $\delta\left(r_{I R C}-r_{C}\right)\left\langle\left|\dot{r}_{I R C}\right|\right\rangle$ using a prelimit form for the Dirac delta (i.e., averaging the value of $\left\langle\left|\dot{r}_{I R C}\right|\right\rangle$ over the samples inside a small range of distance around $\left.r_{C}\right)$ and, at least in principle, using a potential $\mathcal{V}(\mathbf{q})$ computed on the fly by means of $a b$ initio methods. This approach is usually dubbed as efficient microcanonical sampling-transition state theory (EMS-TST). ${ }^{10}$

In spite of its conceptual simplicity, a straightforward MC approach would suffer from a slow convergence of the results due to the low density of samples around $r_{I R C}=r_{C}$ if the internal energy $E$ is low. This problem was already discussed in the literature and two possible solutions were proposed. ${ }^{10,11}$ For instance, Schranz et al. ${ }^{10}$ suggested that a better approach is provided by sampling $[E-\mathcal{V}(\mathbf{q})]^{\alpha}(\alpha$ $<(3 N-5) / 2)$ instead of $W(\mathbf{q})$. This increases substantially the probability of sampling around $r_{I R C}=r_{C}$ and it is possible to correct for the different sampling distribution using the weight $[E-\mathcal{V}(\mathbf{q})]^{(3 N-5) / 2-\alpha}$.

Although formally exact, the introduction of a weight has always the detrimental effect of increasing the variance of the computed quantities and of introducing some bias in their numerical estimate. This may be particularly problematic at low energy, where large weight fluctuations can substantially reduce the signal to noise ratio. To address this shortcoming, Shalashilin and Thompson ${ }^{11}$ proposed to use a kind of importance sampling ${ }^{12}$ (IS) and to estimate the value of the rate constant using the identity

$$
k^{\mathrm{stat}}(E)=\frac{\int_{\Delta \Omega} W(q) d \mathbf{q}}{\int_{\Omega} W(q) d \mathbf{q}} \frac{\int_{\Delta \Omega} W(q) \delta\left(r_{I R C}-r_{C}\right)\left\langle\left|\dot{r}_{I R C}\right|\right\rangle d \mathbf{q}}{\int_{\Delta \Omega} W(q) d \mathbf{q}},
$$

where $\Delta \Omega$ represent an interval of $r_{I R C}$ distances centered around $r_{C}$. The two integrals in Eq. (4) can be estimated separately, and this approach provided a sufficient improvement in the efficiency of the MC sampling to allow the estimate of $k^{\text {stat }}(E)$ for the decomposition of hexahydro-1,3,6-
TABLE I. Statistical rate constants $\left(\mathrm{ps}^{-1}\right)$ for the dissociation of $\mathrm{H}_{5} \mathrm{O}_{2}^{+}$as a function of the internal energy $E(\mathrm{kcal} / \mathrm{mol})$. The headings US(9) and US(3) indicate rate constants computed using umbrella sampling with nine and three windows, respectively. $r_{C}=8$ bohrs in all calculations. The OSS2 dissociation energy for the Zundel cation is $33.27 \mathrm{kcal} / \mathrm{mol}$

\begin{tabular}{rlllc}
\hline \hline & \multicolumn{5}{c}{$k^{\text {stat }}(E)$} \\
\cline { 2 - 5 }$E$ & \multicolumn{1}{c}{ US(9) } & US(3) & \multicolumn{1}{c}{ IS } & EMS-TST $(\alpha=7)$ \\
\hline 38.3 & $0.00051(2)$ & $0.00048(5)$ & $0.002(2)$ & $0.001(2)$ \\
44.6 & $0.0063(2)$ & $0.0073(5)$ & $0.004(2)$ & $0.006(2)$ \\
50.8 & $0.032(1)$ & $0.033(2)$ & $0.036(8)$ & $0.05(1)$ \\
57.1 & $0.098(3)$ & $0.111(6)$ & $0.13(2)$ & $0.10(2)$ \\
63.4 & $0.216(6)$ & $0.26(1)$ & $0.28(3)$ & $0.21(3)$ \\
69.7 & $0.336(8)$ & $0.41(2)$ & $0.39(3)$ & $0.37(3)$ \\
75.9 & $0.51(1)$ & $0.59(2)$ & $0.59(4)$ & $0.63(5)$ \\
82.2 & $0.74(1)$ & $0.85(3)$ & $0.70(4)$ & $0.86(5)$ \\
91.6 & $1.00(2)$ & $1.15(3)$ & $1.04(5)$ & $1.17(6)$ \\
104.2 & $1.38(2)$ & $1.61(4)$ & $1.56(6)$ & $1.50(7)$ \\
\hline \hline
\end{tabular}

trinitro-1,3,5-triazine at low energy (roughly $170 \mathrm{kcal} / \mathrm{mol}$ ). However, it is still possible that the convergence of the first integral in Eq. (4) may be slow if both the critical distance $r_{C}$ and the bond dissociation energy are large. In this case, a large number of samples would be needed.

In the last few years, our group has been interested in studying the energetic and dynamical properties of protonated water clusters $\mathrm{H}^{+}\left(\mathrm{H}_{2} \mathrm{O}\right)_{n}$ using a variety of approaches, ${ }^{13-15}$ and it is currently involved in modeling the low energy cross sections and branching ratios for the reaction between $\mathrm{H}_{3} \mathrm{O}^{+}$and $\mathrm{D}_{2} \mathrm{O}$ extracted from the collisional experiments in Refs. 16 and 17. In this context, a recent attempt of computing the kinetic of the dissociation process for the Zundel cation $\mathrm{H}_{5} \mathrm{O}_{2}^{+}$using statistical reaction theories has highlighted shortcomings in both the MC approaches described previously. ${ }^{10,11}$ In particular, the calculation of $k^{\text {stat }}(E)$ in the range of energies of $10-30 \mathrm{kcal} / \mathrm{mol}$ above the

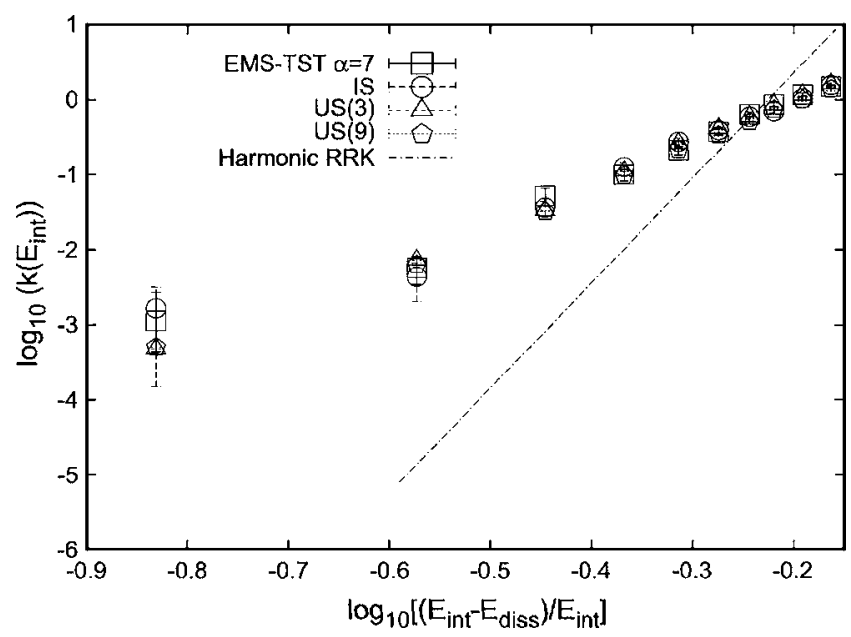

FIG. 2. RRK plot $\left[\log _{10}\left(k^{\text {stat }}\right)\right.$ vs $\left.\log _{10}\left(E-E_{\text {disk }} / E\right)\right]$ of the dissociation rate constants $\left(\mathrm{ps}^{-1}\right)$ for the reaction $\left[\mathrm{H}_{5} \mathrm{O}_{2}^{+}\right]^{*} \rightarrow \mathrm{H}_{2} \mathrm{O}+\mathrm{H}_{3} \mathrm{O}^{+}$computed using Eq. (3) and the methods discussed in the text. US(3) and US(9) indicate simulations carried out using umbrella sampling with either three or nine nested windows, respectively. The dot-dashed line represents the rate constant values computed using classical harmonic RRK theory. 
dissociation threshold was found to be computationally expensive, and it was not possible to properly converge results for total energies in the range of $2-10 \mathrm{kcal} / \mathrm{mol}$ above the threshold. It was found that the causes of this difficulty are the necessity of using a long critical oxygen-oxygen distance $\left(r_{C}\right.$ roughly $8-9$ bohrs) and the large dissociation energy of the Zundel cation (roughly $30 \mathrm{kcal} / \mathrm{mol}$ ).

A new strategy was therefore implemented to reduce the computational cost of the rate constant calculations starting from the similarity between Eq. (3) and the expression for the potential of mean force. To compute the latter, umbrella sampling ${ }^{18}$ (US) is often used to overcome the tendency of the Metropolis method to sample preferentially low energy regions. Similarly, the sampling of $W(\mathbf{q})$ should be "driven" away from the bottom of the PES $\mathcal{V}(\mathbf{q})$. Thus, using ideas presented in Ref. 18, Eq. (3) can be rewritten as

$$
k^{\text {stat }}(E)=\frac{\int_{\Omega} W(\mathbf{q}) \omega\left(r_{I R C}, r_{1}, r_{B}\right)\left\langle\left|\dot{r}_{I R C}\right|\right\rangle d \mathbf{q}}{\int_{\Omega} W(\mathbf{q}) d \mathbf{q}} \frac{\int_{\Omega} W(\mathbf{q}) \omega\left(r_{I R C}, r_{1}, r_{B}\right) \delta\left(r_{I R C}-r_{C}\right)\left\langle\left|\dot{r}_{I R C}\right|\right\rangle d \mathbf{q}}{\int_{\Omega} W(\mathbf{q}) \omega\left(r_{I R C}, r_{1}, r_{B}\right)\left\langle\left|\dot{r}_{I R C}\right|\right\rangle d \mathbf{q}},
$$

where $\omega\left(r_{I R C}, r_{1}, r_{B}\right)=1$ if and only if $r_{1} \leqslant r_{I R C} \leqslant r_{B}$ with $r_{1}<r_{C}<r_{B}$, and zero other way. The function $\omega$, which allows us to select the range of $r_{I R C}$ over which computing expectation values or sampling the microcanonical density, can be accomplished using a square potential with infinite walls. The value of $r_{1}$ is chosen to be larger that the equilibrium distance of the breaking bond, and this first result is similar to the one obtained by Shalashilin and Thompson [Eq. (9), Ref. 11] with a somewhat different choice of the integrands, though. Figure 1 provides a graphical representation of Eq. (5).

Provided that the box function $\omega\left(r_{I R C}, r_{1}, r_{B}\right)$ has a large overlap with the distribution of $r_{I R C}$ obtained sampling $W(q)$, the first ratio of integrals in Eq. (5), henceforth $I_{0}\left(r_{1}, r_{B}\right)$, should be easily computed with good statistical accuracy. The situation might, however, be different for the second ratio, unless the distribution $W(q) \omega\left(r_{I R C}, r_{1}, r_{B}\right)\left\langle\left|\dot{r}_{I R C}\right|\right\rangle$ has a substantial overlap with the region around $r_{I R C}=r_{C}$. If not so, the problem could be alleviated by introducing another distribution $W(q) \omega\left(r_{I R C}, r_{2}, r_{B}\right)\left\langle\left|\dot{r}_{I R C}\right|\right\rangle$ with $r_{1}<r_{2} \leqslant r_{I R C}<r_{C} \leqslant r_{B}$ (see Fig. 1) obtaining

$$
k^{\text {stat }}(E)=I_{0}\left(r_{1}, r_{B}\right) \frac{\int_{\Omega} W(q) \omega\left(r_{I R C}, r_{2}, r_{B}\right)\left\langle\left|\dot{r}_{I R C}\right|\right\rangle d \mathbf{q}}{\int_{\Omega} W(q) \omega\left(r_{I R C}, r_{1}, r_{B}\right)\left\langle\left|\dot{r}_{I R C}\right|\right\rangle d \mathbf{q}} \frac{\int_{\Omega} W(q) \omega\left(r_{I R C}, r_{2}, r_{B}\right) \delta\left(r_{I R C}-r_{C}\right)\left\langle\left|\dot{r}_{I R C}\right|\right\rangle d \mathbf{q}}{\int_{\Omega} W(q) \omega\left(r_{I R C}, r_{2}, r_{B}\right)\left\langle\left|\dot{r}_{I R C}\right|\right\rangle d \mathbf{q}} .
$$

Once again, provided that $W(q) \omega\left(r_{I R C}, r_{1}, r_{B}\right)\left\langle\left|\dot{r}_{I R C}\right|\right\rangle$ has a large overlap with $\omega\left(r_{I R C}, r_{2}, r_{B}\right)$, the first ratio of integrals in Eq. (6), $I_{1}\left(r_{2}, r_{B}\right)$, could be easily estimated by collecting the fraction of number of points falling in the range $r_{2} \leqslant r_{I R C}$ $\leqslant r_{B}$ while sampling $W(q) \omega\left(r_{I R C}, r_{1}, r_{B}\right)\left\langle\left|\dot{r}_{I R C}\right|\right\rangle$. With this choice of sampling distribution, the variance of the integrand is largely reduced and a more precise estimate of the ratio is therefore possible. As for the second ratio in Eq. (6), the overlap between $W(q) \omega\left(r_{I R C}, r_{2}, r_{B}\right)\left\langle\left|\dot{r}_{I R C}\right|\right\rangle$ and the region around $r_{I R C}=r_{C}$ should also be improved because of the reduced volume of configurational space to be sampled. However, if the probability of sampling around $r_{I R C}=r_{C}$ still remains low, the trick leading to Eq. (6) can be used several times until the appropriate sampling is obtained.

The computational scheme provided by Eqs. (5) and (6) was tested by computing the statistical dissociation rates for the Zundel cation $\left(\mathrm{H}_{5} \mathrm{O}_{2}^{+}\right)$as a function of the total internal energy $E$. As mentioned before, these rates represent fundamental quantities if one wishes to model the processes involved in the postcollisional dynamics of $\mathrm{H}_{3} \mathrm{O}^{+}$and $\mathrm{H}_{2} \mathrm{O}$ or in the collision induced dissociation of $\mathrm{H}_{5} \mathrm{O}_{2}^{+}$. Also, they have particular relevance in the discussion of the mechanism of isotopic scrambling when $\mathrm{H}_{2} \mathrm{O}$ is substituted with $\mathrm{D}_{2} \mathrm{O} \cdot{ }^{16,17,19-23}$ The potential energy surface for the simulations was provided by the OSS2 and OSS3 model potentials, ${ }^{24}$ the latter being flexible-polarizable-dissociable potentials known to reproduce with accuracy the dissociation energy of $\mathrm{H}^{+}\left(\mathrm{H}_{2} \mathrm{O}\right)_{n}(n \leqslant 5)$. In this specific case, the dissociation energy is 33.27 and $33.87 \mathrm{kcal} / \mathrm{mol}$ for OSS2 and OSS3, respectively, in good agreement with $33.47 \mathrm{kcal} / \mathrm{mol}$ obtained using QSDCI/6-311++ $\mathrm{G}^{* *}$ in Ref. 16. $4 \times 10^{7}$ points were sampled to estimate each of the integrals needed when employing the methods in Refs. 10 and 11 (i.e., using a grand total of $8 \times 10^{7}$ points for the IS approach). It was also chosen to adopt $\alpha=7$ and $\Delta \Omega=0.8$ for EMS-TST and IS, respectively, as suggested in the original papers. Similarly, the integrals needed for the umbrella sampling [Eqs. (5) and (6)] were estimated using $4 \times 10^{7}$ points each. In the latter case, three $\left[r_{i}=5.0,6.0\right.$, and 7.0 bohrs; US(3) $]$ and nine $\left[r_{i}=5.0,5.5,6.0,6.25,6.5,6.75,7.0,7.25\right.$, and 7.5 bohrs; US(9)] nested sampling windows $\left(\omega\left(r_{I R C}, r_{i}, r_{B}\right)\right)$ were used to evaluate the performance of the method. $r_{C}=8$ and $r_{B}$ $=10$ were used in all cases, the $r_{C}$ value being chosen to roughly minimize the value of the statistical dissociation rates. The blocking method ${ }^{25}$ was employed to eliminate serial correlation between blocks of data. Because the results obtained with the two model potentials were similar, only the OSS2 results are shown in Table I and Fig. 2. The latter also shows the values of the rate constant obtained using classical harmonic RRK theory. ${ }^{3}$ In this case, the second derivatives necessary to estimate the harmonic frequencies were com- 


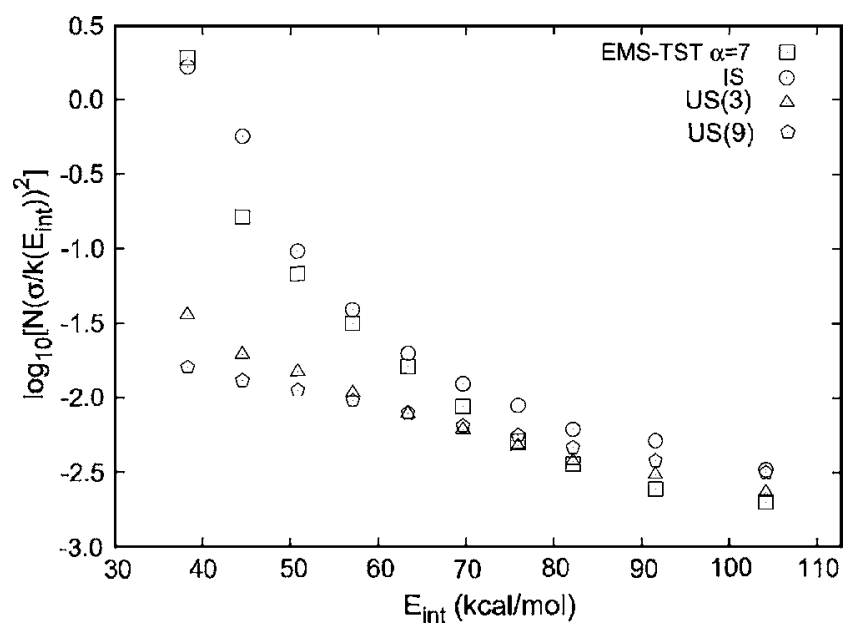

FIG. 3. Plot of the statistical inefficiency $s(E)=\sigma^{2}(E) N /\left(k^{\text {stat }}(E)\right)^{2}$ as a function of the energy $E(\mathrm{kcal} / \mathrm{mol})$ for the MC simulations described in the text. The headings US(9) and US(3) indicate rate constants computed using umbrella sampling with nine and three windows, respectively.

puted using a five-point finite difference scheme.

In presenting the results, we compare the relative efficiency of the three methods in computing the statistical reaction rates for our test case before discussing the relevance of the computed dissociation rates on the modeling of the gas phase reaction between $\mathrm{H}_{3} \mathrm{O}^{+}$and $\mathrm{D}_{2} \mathrm{O}$. As initial remark on the results in Fig. 2, we notice that the three simulation approaches produce results in good agreement at high energy. Small discrepancies are, however, present (see for instance $E=91.6 \mathrm{kcal} / \mathrm{mol}$ ), and these are most likely due to the limited sampling carried out for the IS approach and to a possible bias in the estimator for the rate constant when using EMS-TST. In spite of this, the good agreement strongly suggests that an appropriate stochastic sampling was used and that there should be no problems due to quasiergodicity in the simulations. So, a good conservative estimate of the total error for US(9) should be provided by three times the quoted standard error.

Similar comments can be made for the low energy simulations case, although the statistical error associated with the rate constant estimate is seen to strongly depend on the MC method employed. In particular, the rates computed with EMS-TST and IS at $E=38.3$ and $44.6 \mathrm{kcal}$ have no statistical meaning due to the large standard error associated. Because more points were used in the IS and US simulations than in the EMS-TST ones, the total number of samples should be taken into account in judging the relative efficiency. To make the comparison more transparent, Fig. 3 shows the relative statistical inefficiency $s(E)=\sigma^{2}(E) N /\left(k^{\text {stat }}(E)\right)^{2}$ for the three methods, $N$ being the total number of samples, in units of $10^{7}$, used for each method [e.g., $N=10$ for the US calculations using nine nested windows US(9)]. In the previous equation, $\sigma(E)$ is the standard error for $k^{\text {stat }}(E)$, which could be estimated using the error propagation formula $\sigma^{2}(E)$ $=\left[k^{\text {stat }}(E)\right]^{2} \sum_{i} \sigma_{i}^{2}(E) / I_{i}^{2}$. Here, $\sigma_{i}(E)$ is the standard error associated with $I_{i}$, the value of the $i$ th ratio of integrals [see Eq. (6) and the following discussion]. Alternatively, $\sigma^{2}(E)$ could be estimated by directly simulating the dispersion of its values under the assumption of a Gaussian distribution for all
$\sigma_{i}(E)$. Tests made on preliminary simulations showed these two procedures to be largely equivalent, and it was therefore decided to present only values of $\sigma^{2}(E)$ obtained with the error propagation formula.

From Fig. 3, it is evident that US(9) is far more efficient than the EMS-TST and IS approaches at low energies, providing an improvement of two orders of magnitude in the statistical efficiency of the simulations at energies just above the dissociation threshold. This result translates directly into a two orders of magnitude saving in computational time for a chosen relative standard error. As one should expect, the advantage of the umbrella sampling decreases upon increasing $E$, and eventually EMS-TST $(\alpha=7)$ becomes marginally more efficient for $E \geqslant 75 \mathrm{kcal} / \mathrm{mol}$. The comparison between the IS, US(3), and US(9) results also shows that less windows are necessary to obtain precise results at higher energies.

Comparing the harmonic and $\mathrm{MC}$ results for the dissociation rates clearly indicates that anharmonic behavior has a profound effect on the value of the rate constants. This is most likely due to the long range nature of the interaction potential between the two fragments $\mathrm{H}_{3} \mathrm{O}^{+}$and $\mathrm{H}_{2} \mathrm{O}$, as well as to the known anharmonicity of several vibrational modes of the Zundel cation. This finding is also supported by the number of "effective" degrees of freedom $s$ obtained by fitting the simulation results with the classical RRK formula $k(E)=a\left(\left(E-E_{0}\right) / E\right)^{s-1}$. Indeed, it was found that a $s$ value spanning the range between 6 and 7 gave the best fit for the MC rate constants, a value much smaller than the total number of vibrational degrees of freedom (15) of the Zundel cation. As discussed at length by Song and Hase, ${ }^{26}$ a value of $s$ smaller than the number of harmonic modes does not necessarily indicate a reduced number of active degrees of freedom, but instead simply suggests the failure of the harmonic model in describing the number of accessible states of the system. Song and Hase also provide alternative fitting formulas for which $s$ is not an optimizable parameter and should be chosen equal to the number of vibrational modes. These equations take into account the effect of anharmonicity by means of energy dependent correction terms for the phase space volume and for the position of the critical distance. Unfortunately, our attempt to fit the MC rates in Table I with these improved equations ${ }^{26}$ failed to produce accurate fits if the value of $s$ was constrained to be 15, a finding that deserves further studies.

The results in Table I allow one to obtain an estimate of the average lifetime for the hot Zundel cation. In particular, it is seen that $\mathrm{H}_{5} \mathrm{O}_{2}^{+}$has an average lifetime of roughly $2000 \mathrm{ps}$ when the internal energy is $E=38.3 \mathrm{kcal} / \mathrm{mol}$. For energies closer to the Zundel dissociation threshold, the average lifetime is expected to be even longer, therefore supporting the general idea that proton or deuterium exchange between (for instance) $\mathrm{H}_{3} \mathrm{O}^{+}$and $\mathrm{D}_{2} \mathrm{O}$ at thermal energies takes place via a hot collisional complex $\left(\mathrm{H}_{3} \mathrm{D}_{2} \mathrm{O}_{2}^{+}\right)$lasting several vibrational periods. With such a long lifetime, one would expect that a statistical branching ratio between $\mathrm{H}_{2} \mathrm{DO}^{+}$and $\mathrm{HD}_{2} \mathrm{O}^{+}$should be measured when $\mathrm{H}_{3} \mathrm{O}^{+}$and $\mathrm{D}_{2} \mathrm{O}$ collide with low relative energies. This idea is at variance with the experimental finding provided by Honma and Armentrout ${ }^{16}$ 
and by Anicich and Sen, ${ }^{23}$ who found nonstatistical branching ratios at low energies in their single-collision experimental setups. Although these results were explained by invoking the direct competition between the dissociation and exchange processes taking place after the collision, an idea also supported by RRKM modeling, ${ }^{16}$ it is our view that further investigations on the dynamical processes involved in the hot Zundel cation should be granted to fully clarify this situation.

In conclusion, we presented an adaptation of MC umbrella sampling for the calculation of statistical dissociation rate constants at low energies. Comparing numerical results for the reaction $\left[\mathrm{H}_{5} \mathrm{O}_{2}^{+}\right]^{*} \rightarrow \mathrm{H}_{2} \mathrm{O}+\mathrm{H}_{3} \mathrm{O}^{+}$, umbrella sampling appears to be up to two orders of magnitude more efficient that other literature methods. ${ }^{10,11}$ This substantial improvement in efficiency paves the way for the calculation of low energy dissociation rates for larger systems. Among these, the clusters composed by ions and their molecular ligands [e.g., $\mathrm{H}^{+}\left(\mathrm{H}_{2} \mathrm{O}\right)_{n}$ and $\mathrm{Na}^{+}\left(\mathrm{H}_{2} \mathrm{O}\right)_{n}$ ] are of interest to us. The numerical results also suggest that anharmonicity plays a relevant role in the dissociation process at all energies, a finding deserving particular consideration in modeling the reactive processes involved in the internal dynamics of hot $\mathrm{H}^{+}\left(\mathrm{H}_{2} \mathrm{O}\right)_{n} \mathrm{D}_{2} \mathrm{O}$ complexes.

\footnotetext{
${ }^{1}$ E. Wigner, Trans. Faraday Soc. 34, 34 (1938).

${ }^{2}$ H. Eyring, J. Chem. Phys. 3, 107 (1935).

${ }^{3}$ W. Forst, Theory of Unimolecular Reactions (Academic, New York, 1973).

${ }^{4}$ L. Sun, K. Song, and W. L. Hase, Science 296, 875 (2002).
}

${ }^{5}$ S. C. Ammal, H. Yamataka, M. Aida, and M. Dupuis, Science 299, 1555 (2003).

${ }^{6}$ G. H. Peslherbe and W. L. Hase, J. Chem. Phys. 105, 7432 (1996).

${ }^{7}$ H. W. Schranz, S. Nordholm, and B. C. Freasier, Chem. Phys. 108, 69 (1986).

${ }^{8}$ H. W. Schranz, L. M. Raff, and D. L. Thompson, Chem. Phys. Lett. 171, 68 (1990)

${ }^{9}$ J. W. Brady, J. D. Doll, and D. L. Thompson, J. Chem. Phys. 74, 1024 (1981).

${ }^{10}$ H. W. Schranz, L. M. Raff, and D. L. Thompson, J. Chem. Phys. 94, 4219 (1991).

${ }^{11}$ D. V. Shalashilin and D. L. Thompson, J. Phys. Chem. A 101, 961 (1997).

${ }^{12}$ N. Metropolis, A. W. Rosembluth, M. N. Rosembluth, A. H. Teller, and E. Teller, J. Chem. Phys. 21, 1087 (1953).

${ }^{13}$ M. Mella and D. C. Clary, J. Chem. Phys. 119, 10048 (2003).

${ }^{14}$ M. Mella, D. C. Clary, J.-K. Kuo, and M. L. Klein, Phys. Chem. Chem. Phys. 7, 2324 (2005).

${ }^{15}$ M. Mella and A. Ponti, ChemPhysChem (accepted).

${ }^{16}$ K. Honma and P. B. Armentrout, J. Chem. Phys. 121, 8307 (2004).

${ }^{17}$ S. Yamaguchi, S. Kudoh, Y. Okada, T. Orii, K. Takeuchi, T. Ichikawa, and H. Nakai, J. Phys. Chem. A 107, 10904 (2003).

${ }^{18}$ G. M. Torrie and J. P. Valleau, J. Comput. Phys. 23, 187 (1977).

${ }^{19}$ P. W. Ryan, C. R. Blakley, M. L. Vestal, and J. H. Futrell, J. Phys. Chem. 84, 561 (1980).

${ }^{20}$ D. Smith, N. G. Adams, and M. J. Henchman, J. Chem. Phys. 72, 4951 (1980).

${ }^{21}$ N. G. Adams, D. Smith, and M. J. Henchman, Int. J. Mass Spectrom. Ion Phys. 42, 11 (1982).

${ }^{22}$ M. Henchman, D. Smith, and N. G. Adams, Int. J. Mass Spectrom. Ion Process. 109, 105 (1991)

${ }^{23}$ V. G. Anicich and A. D. Sen, Int. J. Mass Spectrom. Ion Process. 172, 1 (1998).

${ }^{24}$ L. Ojamäe, I. Shavitt, and S. J. Singer, J. Chem. Phys. 109, 5547 (1998).

${ }^{25}$ H. Flyvbjerg and H. G. Peterson, J. Chem. Phys. 91, 461 (1989).

${ }^{26}$ K. Song and W. L. Hase, J. Chem. Phys. 110, 6198 (1999). 